\title{
Increase in gamma band qeeg activity during executive coaching: Some preliminary observations and possible implications
}

\author{
Lyra Puspa ${ }^{1}$, Nurhadi Ibrahim ${ }^{2}$, and Paul T. Brown ${ }^{3}$ \\ ${ }^{1}$ Salomon Centre for Applied Psychology, Faculty of Social and Applied Sciences, Canterbury Christ \\ Church University, Kent, UK; and VanayaNeuroLab Brain \& Behavior Research Center, Jakarta, \\ Indonesia \\ ${ }^{2}$ CityDepartment of Medical Physiology, Faculty of Medicine; Medical Technology Cluster IMERI; \\ and Neuroscience \& Brain Development Cluster IMERI; Universitaslndonesia, Jakarta, Indonesia \\ ${ }^{3}$ andFaculty Professor in Organizational Neuroscience, Monarch Business School, Zurich, \\ Switzerland
}

\begin{abstract}
Executive coaching has become a well-established method of leadership development in many organizations. However, nothing is known about what effects coaching processes have on brain activity in understanding the neurobiological basis of behavioral changes. Using quantified electroencephalogram (qEEG) activity in the gamma-band range, this pilot study is the first attempt to elucidate the electro-cortical patterns that are induced during an executive coaching process whilst using a specific intervention called the CARE Model. Gamma-band oscillation has been related to gestalt perception, the state of consciousness, and cognitive functions. Synchronization of gamma-band has also been proposed as being involved in the mechanisms of functional integration underlying changes in neural circuitry. Despite the decades of gamma-band studies, the possibility that executive coaching might induce gamma-band oscillations has not yet been explored. The subjects were six top talent volunteers (male, mean age $=30$ ). The EEG was recorded from 19 electrodes of the International 10/20 System in both the resting state as the baseline and the continuous 45-minute coaching session. Logarithmically transformed absolute spectral power values of gamma-band were calculated for 3s epochs; and a 182 epochs comparison of each CARE Model stage with the baseline was analyzed using Wilcoxon signed-rank test.Our preliminary qEEG observations show that gamma-band activity became significantly increased in the frontal and posterior regions of the subjects during the coaching process. These preliminary results suggest that the neural impact of executive coaching within the CARE Model may be to evoke a self-reflecting process, gestalt perception, and associative learning.
\end{abstract}

Keywords:executive coaching, leadership, gamma-band, neural oscillation, qEEG, consciousness, gestalt perception. 


\section{Introduction}

In many organizations a good deal of time, money and effort is spent in the personal development of leaders and those with high potential who might become the future leaders. Among many developmental methods, executive coaching has become a well-established method of leadership development in various organizations. However, nothing is known about what effects these developmental processes have on brain activity, and there is at present no neurobiological basis for comparing one developmental method over another. This study reports some preliminary findings that form part of a longer-term study to establish an experimental literature in this field.

Coaching aims to unlock people's potential to maximize their own performance, by helping them to learn rather than teaching them (Whitmore, 2009). The Association for Coaching (AC) defines coaching as "a collaborative solution-focused, results-orientated and systematic process in which the coach facilitates the enhancement of work performance, life experience, self-directed learning and personal growth of the coachee" (AC, 2012). It implies that coaching is an inside-out, peopledevelopment methodology that focuses on behavioral change and personal growth.

Coaching is about facilitating the coachee's change and growth toward desired goals through provoking awareness and responsibility (Whitmore, 2009; Grant, 2012). Hence, despite the differences in methodology used among various schools of thought in coaching, executive coaching process basically consists of four vital parts. First, the clarity of goal (Whitmore, 2009); second, the awakening of the 'aha' or creating a meaningful awareness (Bachirova, 2010); third, deciding the solution and planning for future actions (Cavanagh and Grant, 2010); and fourth, the internal accountability to stay on track (Kauffman et.al., 2010).

All the above four critical parts of coaching were embedded in the development of the CARE Coaching Model as a framework that divides a particular coaching session into four stages: Clarity, Awakening, Resolution, and Empowerment (Puspa, 2016). Clarity is the initial process to facilitate the coachee to discover his/her essential goal and establish the specific objective of the coaching session in order to reach the goal. Awakening is the stage that provides the opportunity for the coachee to unfold his/her self-awareness through mindful reflection. The Resolution stage involves the process of finding the solution and creating an action plan. The last stage in the CARE Model is Empowerment, where the coach helps the coachee to build commitment and accountability for longterm embodiment of the changes gained.

Internal motivation to change and grow as a result of an effective coaching process is induced by evoking self-awareness (Whitmore, 2009); ranging from bare awareness to mindfulness through selfreflecting processes (Cavanagh and Spence, 2013). However, despite the wealth of psychological studies regarding the relationship between executive coaching and awareness, self-reflection, and mindfulness, we still lack of neurobiological evidence that underlies such descriptions.

Meanwhile, studies in neuroscience have revealed that gamma-band oscillation has been related to gestalt perception, the state of consciousness, and cognitive functions. Extensive research on gammaband activity (GBA) using intra-cortical recordings, electroencephalogram (EEG), and magnetoencephalogram (MEG) has demonstrated the importance of this signal in a variety of cognitive processes, ranging from bottom-up-driven perception of gestalt-like or meaningful information to top-down-guided functions such as selective attention, learning, and memory (Kaiser and Lutzenberger, 2003). Studies have also discovered that GBA does not serve a single function, but rather supports multiple cognitive functions including perception, selective attention, memory, motivation, and behavioral control (Bosman, CA. et.al, 2014).

Accumulating evidence shows that meditation practice induces neuroplasticity in self-referential and attentional networks. Mindfulness meditation is observed to increase gamma-band activity in fronto-parietal region, posterior, and parieto-occipital sites during meditation when compared to 
resting state in expert practitioners (Lutz et.al., 2004; Cahn et.al., 2010; Berkovich-Ohana, 2011). These studies suggest that gamma power increases attention, sensory awareness, and self-reflection. Other studies have also shown that GBA may also reflects the formation of percept and associative learning (Miltner, et.al., 1999); the integration of multisensory object representations including the formation of higher-order conceptual perception (Yuval-Greenberg and Deouell, 2007); and the stage of stabilized awakening during meditation wherein the 'ordinary mind' shifts to the 'awakened mind' (Schoenberg, et.al., 2018). The EEG LORETA result of a recent study (Schoenberg, et.al., 2018) demonstrated that the awakening state involved high gamma-band current density within the anterior cingulate cortex (ACC), precuneus, and superior parietal lobule.

However, despite the decades of gamma-band studies, the possibility that executive coaching might induce gamma-band oscillations has not yet been explored. Using the quantitative electroencephalogram (qEEG) activity in the gamma-band range, this pilot study is the first attempt to elucidate the electro-cortical patterns that are induced during an executive coaching process.

\section{Research questions}

This pilot experimental study is the first phase of a planned larger study that is specifically addresses the following questions:

1. Does executive coaching, particularly within the CARE Model framework, activate gamma-band oscillations in the cortical regions?

2. If so, might the gamma-band activity induced by coaching be identified as the underlying neural mechanism of a self-reflecting process to ignite internal awakening?

\section{Method}

\section{Participants}

Six healthy participants (male; right-handed; mean age: 31,6; age range: 30-34) took part in this experiment. They worked at a commercial organization, and were randomly selected as volunteers by their Human Resource (HR) Division among those who are considered to be top talents in the organization. The experiment was approved by the Professional Development Foundation (PDF) on behalf of Canterbury Christ Church University (CCCU) in London, UK and by the Medical Research Ethics Committee, Faculty of Medicine, Universitas Indonesia in Jakarta, Indonesia (No: 0845/UN2.F1/ETIK/2018). Each participant completed their informed consent prior to participating in the experiment.

\section{Design and Procedure}

The exploratory study was conducted using a within-subject experimental design. The brain data was recorded in both the 5-minute resting state (RS) as the baseline and the continuous 45-minute coaching session as the coaching state (CS). Each participant ( $\mathrm{n}=6$ participants) underwent a coaching session using the CARE Coaching Model which is developed by LyraPuspa (Puspa, 2016). Each session within the CARE Model framework was divided into four stages or sub-sessions, namely Clarity, Awakening, Resolution, and Empowerment. This study focuses to analyze the GBA at the Awakening stage, that is assumed to relate with self-reflecting process, awakened awareness, and integrative perception, compared to the baseline.

To ensure that the standardized CARE coaching protocol can produce similar impact whoever is the coach, there were two coaches. Each one was assigned randomly to coach three participants. Both coaches have been trained at the same level of proficiency in using the CARE Model.

\section{Data Recording}


The EEG was recorded from 19 electrodes of the International 10/20 System using DeyMedTruScan EEG along with Deymed EEG Cap to ensure the coachee'scomfortability during data capturing process. The data analyzed used an averaging reference of all electrodes. DeymedTruScan Acquisition software that is embedded with the device was used to monitor the impedance and apply the notch filter $(60 \mathrm{~Hz})$ during recording.

Spontaneous EEG during RS was recorded for $10 \mathrm{~min}(5 \mathrm{~min}$ eyes open and then 5 min eyes closed) prior to the coaching session. This served as the baseline state for comparison with each coaching stage or sub-session within the CARE Coaching Model. During the recording of 45-minute coaching session, markers of each CARE stage were applied to separate each stage of brain data record. The EEG brain data for each stage was then subject to analysis separately from the RS. This study examines especially the GBA of the CS at the Awakening stage of CARE Model.

\section{Data Processing}

The collected brain data were analyzed by using EEGLAB toolbox (Delorme \&Makeig, 2004). It was divided into 3 seconds-epoch. In total, there were 182 artefact-free epochs within both RS and CS to be examined. During pre-processing, filtering was applied ( $1-45 \mathrm{~Hz}$ band-pass filtered). Some other standard pre-processes were also carried out such as bad channel removal, channel interpolation, line noise removal, automatic epoch rejection, and Independent Component Analysis (ICA) to eliminate artefacts and ensure the validity of data.

After multitapering the raw data in the pre-processing stage, the power (measured in $\mu \mathrm{V}^{2}$ ) of gamma-band was transformed from the temporal domain to the frequency domain using the fastFourier transform (FFT) algorithm. For each electrode, the absolute gamma-band power was log transformed and averaged across the 182 epochs. The artefact-free epochs log gamma power was then examined for further analysis.

\section{Statistical Analysis}

After completing the data processing, the cleaned data's frequency-band-powers (i.e. Theta, Delta, Alpha, Beta, and Gamma) for each session were extracted. Each power of each sub-session was compared against the baseline's data of gamma-band power. This comparison was carried out by using Wilcoxon Signed Rank t-test due to the small number of samples that did not meet t-test normality assumptions (Imam, et al., 2014). SPSS (ver.20 for Windows) was utilized to perform the analysis.

\section{Results and Discussion}

\section{Results}

The study revealed that GBA was significantly increased in the CS compared to RS (Fig. 1A). Comparing to RS gamma-power (mean $=0.037 \mu \mathrm{V}^{2}$; SD 0.009; median $=0.0207 \mu \mathrm{V}^{2}$ ), the CS gammapower was more than tripled (mean $=0.104 \mu \mathrm{V}^{2}$; SD 0.022; median $=0.0770 \mu \mathrm{V}^{2}$ ), which may contribute to a very high level of significance $(\mathrm{p}<0.0005)$. There were 18 electrodes that showed positive ranks among 19 electrodes recorded (mean rank $=10.28 ; \mathrm{p}<0.05$ ). The result is described in the following table and chart (Fig. 1).

(A)

\begin{tabular}{|c|c|c|c|}
\hline Band & $\begin{array}{c}\text { Resting State } \\
\left(\mu \mathrm{V}^{2}\right)\end{array}$ & $\begin{array}{c}\text { Coaching State } \\
\left(\mu \mathrm{V}^{2}\right)\end{array}$ & p-values \\
\hline Gamma & $.037 \pm .009$ & $.104 \pm .022$ & $\mathrm{P}<0.0005$ \\
\hline
\end{tabular}


(B)

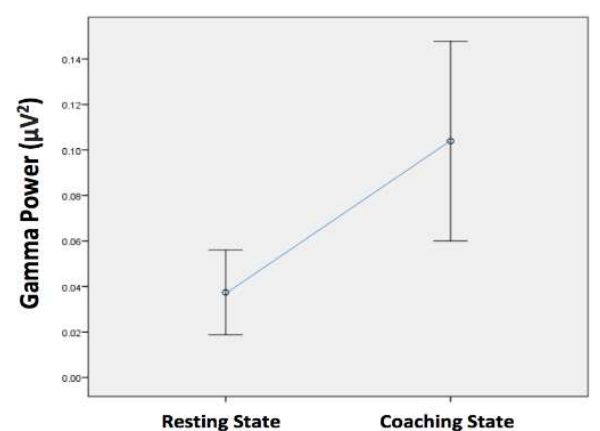

(C)

\begin{tabular}{|ll|r|r|r|}
\hline & Ranks \\
\hline Coaching - Resting & Negative Ranks & $1^{\mathrm{a}}$ & Mean Rank & Sum of Ranks \\
& Positive Ranks & $18^{\mathrm{b}}$ & 10.28 & 5.00 \\
& Ties & $0^{\mathrm{C}}$ & & 185.00 \\
& Total & 19 & & \\
\hline P< $\mathbf{0 . 0 5}$ (Significant)
\end{tabular}

Fig. 1. (A) Overall log gamma-band power (Mean \pm SE) during CS and RS ( $p<0.0005$; Wilcoxon paired-rank t-test); (B) The visual comparison of overall gamma-band power between RS vs CS (Mean \pm SE); and (C) Positive (increased) vs negative (decreased) changes of GBA within all 19 electrodes ( $\mathrm{p}<0.05$; Wilcoxon paired-rank t-test).

Among 19 electrodes observed, the gamma-band power was significantly increased in 14 electrode channels that were spread within the frontal (Fp1, Fp2, F7, Fz, F4, F8), temporo-parietal (T5, T6), right central (C4), and parieto-occipital (P3, Pz, P4, O1, O2) regions. The neural pattern of GBA revealed in this study is similar to the previous study of GBA related self-reflecting process (Lutz et.al., 2004; Cahn et.al., 2010). This data supports a positive answer to the second research question.

There is no significant increase of GBA in the left superior frontal (F3), left central (C3, Cz), and bilateral temporal $(\mathrm{T} 3, \mathrm{~T} 4)$ regions. The log gamma-band power and $\mathrm{p}$ value of significantly activated regions are presented in the following table and brain map.

\begin{tabular}{|c|c|c|c|}
\hline Channel & Resting State & Coaching State & p-values \\
\hline Fp1 & $.011 \pm .002$ & $.033 \pm .009$ & .028 \\
\hline Fp2 & $.016 \pm .003$ & $.054 \pm .010$ & .028 \\
\hline F7 & $.047 \pm .019$ & $.162 \pm .045$ & .028 \\
\hline F3 & $.021 \pm .003$ & $.069 \pm .036$ & .116 \\
\hline Fz & $.012 \pm .002$ & $.019 \pm .002$ & .028 \\
\hline F4 & $.029 \pm .010$ & $.110 \pm .037$ & .028 \\
\hline F8 & $.060 \pm .021$ & $.299 \pm .112$ & .028 \\
\hline T3 & $.142 \pm .061$ & $.153 \pm .039$ & .917 \\
\hline C3 & $.056 \pm .036$ & $.045 \pm .005$ & .345 \\
\hline Cz & $.013 \pm .003$ & $.020 \pm .004$ & .141 \\
\hline C4 & $.038 \pm .011$ & $.145 \pm .032$ & .028 \\
\hline T4 & $.146 \pm .053$ & $.378 \pm .179$ & .463 \\
\hline T5 & $.022 \pm .005$ & $.117 \pm .022$ & .028 \\
\hline P3 & $.015 \pm .003$ & $.030 \pm .002$ & .046 \\
\hline Pz & $.010 \pm .001$ & $.020 \pm .002$ & .028 \\
\hline P4 & $.016 \pm .004$ & $.044 \pm .006$ & .028 \\
\hline T6 & $.021 \pm .002$ & $.114 \pm .028$ & .028 \\
\hline O1 & $.017 \pm .002$ & $.086 \pm .010$ & .028 \\
\hline
\end{tabular}

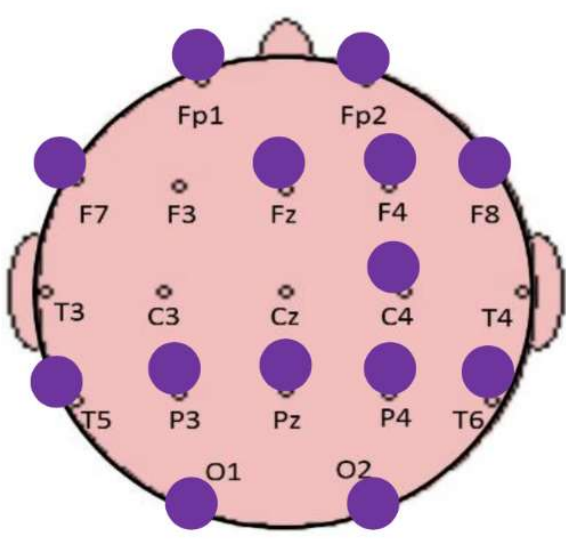

Fig. 2. (A) Log gamma-band power (Mean $\pm \mathrm{SE}$ ) and individual $\mathrm{p}$-values of each electrode channel during RS and CS (GBA significance is observed in 14 electrode channels that have pvalues in bold numbers using $\mathrm{p}<0.05$; Wilcoxon paired-rank t-test); (B) The neural correlates of significant GBA in the stimulated 14 activated electrodes $(\mathrm{p}<0.05)$. 
The brain map in Fig. 2 shows that the spread of GBA induced was proved to be more stimulated in the right hemisphere, as observed in the increase of GBA in right hemispheric neural correlates (Fp2, F4, C4, P4, O2) and the absence of significant increase of GBA in left superior frontal (F3) site.

\section{Discussion}

These preliminary results reveal that executive coaching, in particular the Awakening stage of CARE Model, significantly induces gamma-band oscillations at a number of brain regions. It suggests that coaching may be considered as an effective method to ignite the awakening state of mind. What the coaches did in the Awakening stage of CARE Model was a deliberate thought-provoking process to facilitate the bottom-up self-awareness intended to evoke the 'Aha' moment through reflection and an inner-conversation process (Puspa, 2016). This stage is critical to stimulate the internal drive to change. The preliminary findings of this study are aligned with the extensive studies of GBA which showed the role of gamma-band in relation with self-awareness, self-reflecting process, and internal motivation (Bosman, CA. et.al, 2014; Yuval-Greenberg and Deouell, 2007; Schoenberg, et.al., 2018).

The increase of GBA in parieto-occipital and temporo-parietal regions in this study also provides an indication that meaningful insight as a result of coachee's self-report in Awakening stage of CARE Model may be related with the integrative gestalt perception and associative learning as in previous scientific evidence (Kaiser and Lutzenberger, 2003; Bosman, CA. et.al, 2014). The coaching questions in the Awakening stage of CARE Model such as, "Why is it important to you?" or "What does it mean for you?" seemed to stimulate the associative gestalt thinking to connect each visual mental image to form a new meaning.

Considering the similarity of gamma-band pattern within the frontal, posterior, and parietooccipital in this study and previous mindfulness meditation studies (Lutz et.al., 2004; Cahn et.al., 2010; Berkovich-Ohana, 2011), it is also a possibility that executive coaching may induce similar state of mindfulness as in meditation practices. This preliminary indication is highly interesting, since executive coaching requires a 1on1 continuous conversation with the presence of a coach in the process, whereas meditation involves a succession of solitary moments. Further research should be conducted to compare the neural mechanism of coaching and meditation as mindfulness induction methodologies; and how such internal mechanism could contribute to the development of leadership and personal development capacity.

However, as an initial part of a planned larger scale research, the preliminary result of this pilot study should be considered as essentially indicative. Further research is needed to examine whether a larger sample may result in similar findings. The inclusion of females, non-talents, and a broader range of age in future experiment should be involved to substantiate or question whether these results that can be generalized, as would a study in which normal conversation was employed without a specific focus on change. It will also be interesting for further experimental work to cover all stages of the CARE Model and all frequency bands to capture more comprehensive insights. Since gamma-band synchronization has also been proposed as being involved in the mechanisms of functional integration that may induce short-term and long-term neural changes (Miltner, et.al., 1999; Doesburg, et.al., 2008), further studies should also be conducted to explore whether coaching is also an effective stimulation of the formation of Hebbian assemblies of cells whose synaptic linkages are strengthened and may cause changes in the physical structure of the brain.

\section{Conclusions}

Our preliminary qEEG observations during executive coaching show that gamma-band activity became significantly increased in the frontal and posterior regions of the subjects during the coaching process. These preliminary results suggest that the neural impact of executive coaching within the 
CARE Coaching Model may be to ignite self-reflection, gestalt perception, awakened awareness, associative learning, and mindfulness state.

Further research is needed to assess what neural patterns might appear across all coaching stages; what long-term effects might persist; what results from broader range of participants; what effects are produced by specific variations in the content of coaching; and whether coaching is also an effective stimulation of the neuronal cell assembly process that may lead to structural neural changes.

\section{Acknowledgments}

This paper is based on the small-scale research as an initial part of a larger scale experiment of a Ph.D. dissertation (first author), supervised by the second and third authors. The authors are thankful to IhshanGumilar and DhioDelinanda Putra from VanayaNeuroLab for their technical support. This work was supported by VanayaNeuroLab Brain and Behavior Research Center, Indonesia; Indonesian Medical Education and Research Institute (IMERI) Universitas Indonesia; and DeyMed Technology, Czech Republic.

\section{References}

Association for Coaching (AC). (2012). AC Coaching Competency Framework. Retrieved December, 10, 2017 from, https://www.associationforcoaching.com/

Bachirova, T., Cox, E., Clutterbuck, D. (2010). The Complete Handbook of Coaching. London: SAGE Publication Ltd.

Berkovich-Ohana, A., Glicksohn, J., and Goldstein, A. (2011). Mindfulness-Induced Changes in Gamma Band Activity - Implications for The Default Mode Network, Self-Reference and Attention. Clinical Neurophysiology, 123(2012), 700-710.

Bosman, CA., et.al. (2014). Functions of Gamma-Band Synchronization in Cognition: From Single Circuits to Functional Diversity Across Cortical and Subcortical Systems. European Journal of Neuroscience, 39, 1982-1999.

Cavanagh, M.J. and Grant, A.M. (2010). The Solution-Focused Approach to Coaching. In The Complete Handbook of Coaching (pp 54-67). London: SAGE Publication Ltd.

Cavanagh, MJ. and Spence, GB. (2013). Mindfulness in Coaching: Philosophy, psychology or just a useful skill? In The Wiley-Blackwell Handbook of the Psychology of Coaching and Mentoring (pp. 112134), $1^{\text {st }}$ edn. Hoboken, NJ: John Wiley \& Sons, Ltd.

Delorme, A., Makeig, S. (2004). EEGLAB: an open source toolbox for analysis of single-trial EEG dynamics. Journal of Neuroscience Methods, 134:9-21.

Doesburg, SM., et.al. (2008). Large-Scale Gamma-Band Phase Synchronization and Selective Attention. Cerebral Cortex, 18(2008), 386-396.

Grant, A.M. (2012). An Integrated Model of Goal-Focused Coaching: An Evidence- Based Framework for Teaching and Practice. International Coaching Psychology Review, 7(2), 146-165. 
Imam, A., Mohammed, U., and Moses-Abanyam, C. (2014). On Consistency and Limitation of Paired T-Test, Sign and Wilcoxon Sign Rank Test. IOSR Journal of Mathematics, 10(1), 01-06.

Kaiser, J and Lutzenberger, W. (2003). Induced Gamma-Band Activity and Human Brain Function. The Neuroscientist, 9(6), 475-484.

Kauffman et.al. (2010). The Positive Psychology Approach to Coaching. In The Complete Handbook of Coaching (pp 158-171). London: SAGE Publication Ltd.

Lutz A., et.al. (2004). Long-Term Meditators Self-Induce High-Amplitude Gamma Synchrony During Mental Practice. The National Academy of Sciences of the USA, 101(46), 16369-16373.

Miltner, WHR., et.al. (1999). Coherence of gamma-band EEG activity as a basis for associative learning. Nature, 397(1999), 434-436.

Puspa, L. (2016). Coaching Style Leadership: Transformational Leadership with CARE Coaching Model, $1^{\text {st }}$ edn. Jakarta: Vanaya Coaching International.

Schoenberg, PLA., et.al. (2018). Mapping Complex Mind States: EEG Neural Substrates of Meditative Unified Compassionate Awareness. Consciousness and Cognition, 57(2018), 41-53.

Whitmore, J. (2009). Coaching for Performance: GROWing Human Potential and Purpose, 4th edn. London: Nicholas Brealey Publishing.

Yuval-Greenberg, S and Deouell, LY. (2007). What You See Is Not (Always) What You Hear: Induced Gamma Band Responses Reflect Cross-Modal Interactions in Familiar Object Recognition. J. Neurosci, 27(5), 1090-1096. 